Slavica

bruxellensia

\section{Slavica bruxellensia}

Revue polyphonique de littérature, culture et histoire

slaves

$3 \mid 2009$

Pensée des hommes

\title{
La nation dans les conceptions messianiques de Michal Miloslav Hodža
}

\section{Joanna Goszczyńska}

Traducteur : Jeremy Lambert

\section{OpenEdition \\ Journals}

Édition électronique

URL : http://journals.openedition.org/slavica/267

DOI : 10.4000/slavica.267

ISSN : 2034-6395

\section{Éditeur}

Université libre de Bruxelles - ULB

Édition imprimée

Pagination : 7-16

ISSN : 2031-7654

\section{Référence électronique}

Joanna Goszczyńska, «La nation dans les conceptions messianiques de Michal Miloslav Hodža »,

Slavica bruxellensia [En ligne], 3 | 2009, mis en ligne le 15 juin 2009, consulté le 01 mai 2019. URL

http://journals.openedition.org/slavica/267 ; DOI : 10.4000/slavica.267

\section{Ce document a été généré automatiquement le 1 mai 2019.}

\section{(c) (i) (9)}

Les contenus de Slavica bruxellensia sont mis à disposition selon les termes de la Licence Creative Commons Attribution - Pas d'Utilisation Commerciale - Pas de Modification 3.0 France. 


\title{
La nation dans les conceptions messianiques de Michal Miloslav Hodža
}

\author{
Joanna Goszczyńska
}

Traduction : Jeremy Lambert

\section{NOTE DE L'ÉDITEUR}

La langue originelle de cet article est le polonais

Dans les années 1930, Milan Pišút (1908-1984) présenta "Slovenské iskrice » (L'étincelle slovaque, 1856), poème jusqu'alors inconnu de Samo Bohdan Hroboň (1820-1894), en le plaçant dans le contexte du messianisme. Le critique et historien de la littérature slovaque de renom voulait ainsi exprimer que le messianisme slovaque loin d'être un «extrémisme poétique » avait en réalité imprégné toute une génération et lui avait fourni un soutien spirituel fondamental à une époque de magyarisation accrue ${ }^{1}$. Durant de longues années, ce point de vue ne fut pas partagé. La critique slovaque, de la fin du XIX siècle jusqu'aux années soixante du vingtième siècle, l'a soit ignoré volontairement, soit déprécié car elle considérait toute manifestation de la pensée messianique comme idéologiquement inutile. Le messianisme, qui, à la lumière des études actuelles, apparaît comme un élément constitutif du romantisme slovaque, était perçu dans le meilleur des cas comme un phénomène périphérique. Toutefois, il n'était pas rare qu'il fût également perçu comme un phénomène pathologique, comme une tumeur sur le corps sain de la littérature de la renaissance nationale ${ }^{2}$. Selon les thèses les plus courantes, reprises par les générations successives de critiques, la poésie messianique était non seulement incompréhensible, prophétique et visionnaire, mais également détachée des réalités et des besoins de la nation. La prise de conscience de ces besoins nationaux ne pouvait trouver un écho que dans la "poésie slave», telle que la concevait Ludovit štúr 
(1815-1856), le principal idéologue de la renaissance nationale slovaque, qui basait ses conceptions sur la mythification du folklore.

\section{Les messianistes slovaques}

2 Ce diagnostique étonnant peut être partiellement expliqué, nous osons l'affirmer par la mauvaise connaissance des textes, restés pour la plupart à l'état d'archives (certains le sont d'ailleurs encore) $)^{3}$. La lecture de ces textes démontre en effet quelque chose de tout à fait différent. Les poésies des deux plus importants représentants du messianisme slovaque, Hroboň et Michal Miloslav Hodža (1811-1870), proposent non seulement un diagnostique de la réalité de l'époque, mais également des propositions concrètes pour résoudre les problèmes de nationalité. Quelque soit la manière avec laquelle ces conceptions on pu être jugées, il est indéniable qu'elles constituaient une voix importante dans les discussions de l'époque sur le thème de la place et du rôle des Slaves en Europe. Les rédacteurs des journaux contemporains l'avaient d'ailleurs très bien compris : s'ils ne permettaient pas que ces textes fussent imprimés, c'était non seulement parce que ceuxci étaient, selon eux, incompréhensibles mais également, ce dont ils ne se cachaient pas, à cause de leur orientation clairement panslaviste, ce qui était le cas des poèmes de Hroboň par exemple ${ }^{4}$. C'est autant la langue parfois hermétique de ces textes que la peur de la censure qui rendirent impossible leur intégration dans le circuit littéraire. D'autant plus qu'après l'écrasement des révolutions du Printemps des Peuples, à l'époque du régime néo-absolutiste d'Alexander Bach, la censure se faisait intransigeante sur la question du panslavisme dont elle empêchait toute manifestation.

Hroboň eut finalement plus de chance que Hodža. Quelques-unes de ses œuvres furent publiées dans les colonnes des journaux de l'époque, d'autres, comme "Slovenské Iskrice ", dont il a été fait mention plus haut, parurent durant l'entre-deux-guerres. Elles n'éveillèrent cependant pas un grand intérêt, hormis celui de Pišút. Quelques sérieux recueils d'œuvres de Hroboň, ainsi que sa correspondance et des fragments de son journal, furent publiés dans les années 1950 . Sa poésie commença alors à susciter l'intérêt d'un plus grand nombre de lecteurs et à être commentée par les chercheurs. On a même commencé à voir en lui un précurseur des principes tardifs du surréalisme. Hroboň, auparavant considéré comme un poète périphérique, excentrique et oublié, prit une position centrale dans le canon de la littérature romantique slovaque.

L'œuvre de Hodža connut un sort bien moins enviable. Il développa son idée messianiste dans deux de ses compositions: la première intitulée "Matora $»^{5}$ (qu'il produisit entre 1853 et 1857) ne fut publiée intégralement qu'en 2003; l'autre, "Vieroslavin» (écrite dans les années 1867-1870), enrichit encore actuellement les archives slovaques. Il est intéressant de souligner que, dans le poème " Matora », Hodža tenta d'allier l'inspiration folklorique à l'idéologie messianique en faisant du brigand Juraj Jánošik, héros folklorique célèbre, un Messie slovaque. À l'époque, le mythe de Jánošik était enraciné tant dans les textes littéraires que dans la conscience collective. L'utilisation de ce portrait littéraire était presque une obligation tacite des adeptes de l'art scriptural. Entre parenthèses, notons que ce ne fut pas sans être encouragé par Štúr qui postulait expressis verbis le recours aux héros populaires. Jánošik, élevé au rang de héros national par la littérature d'avant le Printemps des Peuples, devint un idéologème attrayant pour les créateurs qui voulaient faire coïncider les idées messianiques avec les inspirations folkloristes ${ }^{6}$. Un autre poète slovaque, Ján Botto (1829-1881), suivra la même voie quelques années plus 
tard. Son poème «Smrǐ Jánošiková » (La Mort de Jánošik, 1862) devint un classique du romantisme slovaque. Cependant dans le poème de Botto, fortement ancré dans la tradition populaire, les liens avec les idées messianistes sont dissimulés derrière l'héroïsation du brigand qui est traité comme un modèle national, porteur de l'idée de liberté. Ce sont ces aspects du poème sur lesquels la critique s'attarda, dépréciant la trame messianique. Cela explique la popularité qu'acquit le poème et le rang exceptionnellement élevé auquel il s'éleva aux yeux des commentateurs. Le lien qui unit le Jánošik de Hodža à celui des récits populaires est plutôt convenu : le poète le présente avant tout comme une incarnation de l'idée messianiste ainsi que comme une relecture de celle-ci. Jánošik, en tant que sauveur potentiel de la nation, subit en effet une défaite totale, il n'est pas en mesure de réaliser les hauts espoirs qui sont placés en lui, il ne peut mener sa nation au combat, et il n'est plus capable non plus d'incarner durablement l'humilité et la souffrance. Il se dégage de la protection divine et retourne à l'incarnation satanique du brigand. Le brigandage est en effet perçu négativement dans le poème de Hodža, au contraire des textes folkloriques. Jánošik est traité ici comme un criminel, un brigand malfaiteur qui pactise avec le diable; il doit conclure une alliance avec Dieu et expier ses erreurs pour mériter sa mission, qui consiste à sauver la nation. En cela, il s'inscrit dans un mode contraire à celui des aventures populaires, qui l'héroïsent complètement, et de la majorité des textes qui construisent le mythe du héros national.

5 La défaite de Jánošik n'est pas uniquement la débâcle de l'attitude messianique. Il est également la manifestation du manque de foi en la possibilité de transformer la nation slovaque dont le représentant est un bandit. Par endroits, l'auteur soumettait sa nation à une critique si sévère qu'il n'est pas étonnant que l'on ne se fut pas empressé de publier son poème. Hodža remit en question le mythe national de la mission historique des Slovaques considérés comme une nation choisie, mythe crée par Štúr et répandu surtout avant 1848. Il présenta en effet une vision de sa nation dans laquelle celle-ci était poussée par l'histoire au plus bas échelon de la hiérarchie des nations. Au reproche de dénationalisation et de dégradation de la langue nationale vinrent s'ajouter des accusations d'un autre ordre. Hodža accusait également les slovaques d'être une nation à l'âme soumise, d'être des serviteurs coincés dans un esclavage vers lequel les pousse leur nature.

\section{" Matory » et "Vieroslavina », deux poèmes messianiques de Hodža}

Le jugement porté sur la nation est l'un des motifs les plus importants des poèmes "Matory " et "Vieroslavina», il s'exprime dans les problématiques de la possibilité laissée à la nation d'exister dans l'histoire et du rôle qu'elle doit tenir sur le territoire européen. Dans les deux poèmes, l'attitude de Hodža est caractérisée par une antinomie. Il semble pourtant que les sources de celle-ci soient différentes dans les deux poèmes.

7 Hodža mit plusieurs années à produire « Matora », ce qui n'est pas surprenant au vu des quelques 20000 vers que compte le poème (soit environ 600 pages). La vision de la nation qui s'en dégage est confuse, elle comporte de nombreuses incohérences et contradictions. Elle témoigne des luttes et des hésitations du poète qui ne croyait plus au succès du mouvement national. Hodža écrivit son poème à une époque de frustration durant laquelle le sentiment de défaite était très présent. Cette ambiance était propice au 
développement d'une pensée messianique. La foi qu'avaient placés les Slovaques en l'Autriche durant la révolution de 1848-1849 et leur association durant cette période s'étaient en effet avérées être des erreurs historiques ${ }^{7}$. Hodža n'était pas un observateur impartial mais véritablement un activiste engagé dans les affaires nationales. Et ce, dans plusieurs domaines ${ }^{8}$. En 1848 , il s'engagea directement dans la création d'une division de fantassins slovaques, soumise à l'État-major autrichien, et prit part à la première expédition menée contre les Hongrois. Elle ne se termina pas de manière glorieuse pour lui puisqu'il quitta la division après la première échauffourée, ce qui lui valut de passer devant un tribunal militaire pour désertion. Cela ne freina toutefois pas son engouement : il continua à participer à la préparation de ce que l'on appelle les «insurrections slovaques » contre les Hongrois. Le gouvernement hongrois de Lajos Kossuth ordonna son arrestation et publia un mandat d'arrêt contre lui (tout comme il l'avait fait contre les autres meneurs des insurrections, Štúr et Hurban). Les autorités de Vienne l'honorèrent néanmoins en lui accordant en 1850 la Croix d'Or du mérite de l'empereur FrançoisJoseph $\mathrm{I}^{\mathrm{er}}$.

8 La politique des Slovaques se révéla imprévoyante et inefficace. La politique autrichienne ne résultait pas de ses obligations envers les nations slaves qui la constituaient, bien au contraire. Le régime mis en place par Bach écrasa les mouvements nationaux et culturels, augmenta la censure et réprima les activistes nationalistes les plus actifs (ou en « normalisa » une partie en leur offrant des postes bien rémunérés dans l'administration). Pour Hodža comme pour beaucoup d'autres hommes qui avaient pris part à la vie nationale, le régime néo-absolutiste furent une période de défaitisme, de perte des illusions et de la foi en la possibilité de relever une nation qui avait laissé passer sa chance. Dans le cas de Hodža, cela se traduisit par une révision des conceptions antérieures ayant pour objet la nation et par une démythification de la vision romantique selon laquelle la nation slovaque avait un rôle particulier à jouer dans l'histoire.

Hodža situait la nation slovaque au bas de l'échelle des nations slaves et ne lui fit par conséquent jouer aucun rôle dans l'histoire, moins encore celui de nation choisie. Il soulignait en effet qu'une nation qui se noie dans un élément étranger perd la chance de renaître. À ce jugement négatif s'alliait pourtant une tentative de justification, elle-même associée à la volonté de rejeter une partie de la responsabilité sur l'autre, sur l'étranger, c'est-à-dire sur les Hongrois qui vivaient sur le même territoire. Hodža les accusait d'ôter sa mémoire historique au peuple slovaque, de ridiculiser et de déformer ses traditions qui légitimaient pourtant son droit à la différenciation identitaire. Il évoquait par exemple la légende, répandue par l'historiographie hongroise, de la soumission des terres slovaques par Svatopluk Ien échange d'un cheval blanc à l'harnachement d'or. Il reprochait à ce fait légendaire, dont la représentation picturale était accrochée aux murs de nombreuses maisons de Hongrois et de Slovaques magyarisés, d'être une falsification de la mémoire historique.

10 Il faut souligner que Hodža ne revendiquait pas pour les Slovaques le droit de se déclarer nation historique. Il affirmait cependant que le fait qu'ils n'étaient pas une nation politique, établie dans les limites d'un état, ne gageait en rien leurs aspirations nationales. Mais il considérait également que les slovaques, par leur politique conciliatrice envers l'Autriche et l'alliance de leur destin à celui de celle-ci lors de la révolution, avaient eux-mêmes enterré leur espoir de voir se réaliser leur programme national qui, comme le remarque Miroslav Hroch dans ses études sur les phases des mouvements nationalistes, peut passer pour un programme politique de substitution? ${ }^{9}$. 
11 Il semble que déjà avant que n'éclate la révolution Hodža n'estimait pas l'Autriche outre mesure. Lorsqu'il apprit, en juillet 1848, que les Hongrois cherchaient à s'entendre avec les nations slaves qui peuplaient le royaume de Hongrie et qu'ils s'apprêtaient à arrêter le principe de leur égalité, le poète ne cacha pas qu'il considérait cette option plus proche de ses idées que celle qui consistait à pactiser avec les dirigeants viennois. Il se rapportait avec un certain scepticisme à cette issue des événements car il ne voyait pas d'autre option que le conflit armé ${ }^{10}$. Notons qu'en juillet 1849, la position des Hongrois, plutôt désunis sur les questions nationales au début de la révolution, se modifia sensiblement: ils publièrent en effet un communiqué garantissant des droits élargis aux différentes nations du royaume de Hongrie. Mais la situation était déjà trop avancée pour que le cours des événements puisse être arrêté.

12 La manière dont le poète slovaque juge la situation dans les années 1850 est l'expression d'un sentiment de désespoir et d'inutilité. Hodža ne voit plus de perspectives pour le mouvement national écrasé, il cherche donc dans l'individualité le secours à apporter à sa nation, il transporte le problème de la lutte pour les droits nationaux sur le terrain du messianisme individuel. Cette voie lui semble la plus évidente, car le héros de " Matora ", le brigand Jánošik, ne se réalise pas en tant que Messie. La croyance messianique en un plan divin concernant l'Histoire est elle aussi discréditée.

Le poète propose une version révisée de ses idées sur les possibilités de sa nation dans sa dernière œuvre, son testament poétique comme il l'appelle lui-même, le poème (jamais publié) intitulé "Vieroslavin ${ }^{11}$, titre que l'on pourrait traduire très librement par " glorification de la foi ». Il l'écrivit à Cieszyń, ville située alors en Autriche, où il passa les trois dernières années de sa vie dans un exil volontaire, qu'il avait accepté en échange d'une retraite hautement rémunérée par l'État. Le prix à payer fut son engagement à ne plus agir publiquement, ce qui signifiait son retrait de la vie nationale.

14 La question de la valeur de sa propre nation pose problème au poète. Celui-ci tente cette fois d'inscrire le développement de sa nation dans un schéma messianique de l'histoire. Sa conception historiosophique se base sur une vision en trois temps de l'Histoire, vision répandue à l'époque romantique, on la retrouve par exemple dans le Livre de la nation Polonaise d'Adam Mickiewicz (1799-1855) ou dans l'historiographie d'Andrzej Towiański (1799-1878). Soulignons que l'œuvre de Mickiewicz, tout comme l'action de Towiański, étaient connus du milieu romantique slovaque. Conformément à cette vision en trois temps, la nation doit passer par les étapes de la chute, de la destruction et enfin de l'expiation et de la renaissance.

15 Hodža situait la chute de la nation slovaque à l'époque de la Grande-Moravie, c'est-à-dire aux temps mythiques de sa création. C'est alors que commença, après une courte période fastueuse, un millénaire d'esclavage qui constitue le deuxième maillon de l'Histoire. À cette époque, la nation subit, dans le domaine politique, une destruction systématique conduite par les Hongrois qui limitaient les droits linguistiques des Slaves et ne reconnaissaient pas le rôle de ceux-ci dans la mise en place d'un état commun, s'en remettant à eux avec hauteur et dédain comme à des représentants d'une nation plébéienne. Le poète slovaque faisait allusion à une expression qui s'était enracinée dans la conscience collective et qui affirmait que « Tot nem ember ", " le Slovaque n'est pas un homme $»^{12}$. Nous nous servons ici d'une conception nationale que nous associons à Hodža, mais nous nous devons de souligner que cette idée était déjà largement répandue avant 
cette étape de développement de la communauté ethnique slovaque. Ajoutons encore que le mythe de l'esclavage millénaire, très fortement ancré dans la conscience collective slovaque, est aujourd'hui encore l'un des mythes politiques les plus utilisés dans la sphère publique ${ }^{13}$.

La disparition de leur nation avait été favorisée par l'attitude des Slovaques eux-mêmes. Hodža incriminait les représentants de toutes les classes sociales, du peuple soumis jusqu'au clergé, en passant par la bourgeoisie et la noblesse. La bourgeoisie parce qu'elle s'était germanisée et la noblesse parce qu'elle s'était magyarisée, ce qui eut pour conséquence que ces deux classes s'étaient d'elles-mêmes éliminées de l'univers national ; le clergé parce qu'il avait perdu son contact avec Dieu et n'avait pas mis en pratique les décrets divins. Selon le poète, cet état de fait était toutefois justifié. La nation slovaque suivait le plan de la Providence, elle avait en effet été nommée, choisie pour souffrir durant mille ans. Cette période d'esclavage, de "Châtiment", était une condition essentielle à sa renaissance future. Sans elle, la nation slovaque ne pourrait pas prendre une place exceptionnelle dans l'histoire de l'humanité. Cette place exceptionnelle serait partagée par les Slovaques et les autres Slaves. Les Péchés, les tourments avaient une signification supérieure, le mal donnerait naissance au bien tandis que de la douleur viendrait la grandeur.

L'idée selon laquelle les Tatras étaient le berceau des Slaves et les Slovaques la plus ancienne nation formée par ceux-ci était enracinée dans la conscience de la génération romantique slovaque. On la rencontrait dans la quasi-totalité des conceptions missionistes, elle permettait de légitimer l'idée selon laquelle les Slovaques avaient été prédestinés à l'importante mission historique qu'ils devaient remplir. Dans son historiosophie messianiste, Hodža introduit toutefois un nouvel élément, en accord avec l'idée même de messianisme: c'était le fait d'avoir souffert durant mille ans qui prédestinait les Slovaques à être une nation choisie. Le poète soulignait que les Slovaques avaient souffert pour les autres nations slaves, que c'était une souffrance voulue par Dieu et que ce sera également par Sa volonté que l'antique gloire reviendra à tous les Slaves. La renaissance des nations slaves était cependant la condition sine qua non de la réalisation des plans divins : elles devaient réaliser la mission divine qui leur avait été assignée afin que les volontés de Dieu soient exaucées. Cette mission était, dans le projet de Hodža, la construction d'une communauté de nations européennes basée sur la foi dans le Christ.

18 Sa vision d'une Europe commune étonne aujourd'hui par son actualité. Hodža voyait l'Europe commune comme la patrie idéale de toutes les nations, germaniques, romanes et slaves, qui la peuplaient. Cette communauté devait être basée sur l'idée d'unification, de compréhension mutuelle et de respect des différences. Les valeurs chrétiennes étaient les fondements sur lesquels elle devait reposer. C'était ces valeurs qui définissaient l'identité européenne commune.

19 Cette vision globalisante doit cependant être nuancée. Le projet de Hodža ne se limitait en effet pas à décrire une union supraéthnique de communautés nationales basée sur le principe du bon vouloir. À l'intérieur de cette large communauté, le poète distinguait un territoire plus petit, celui de l'Autriche, qui devait lier, unir les nations slaves. Il voyait en l'Autriche chrétienne la garante de l'aplanissement des conflits et du maintien des différences nationales.

Dans cette œuvre, l'auteur exprimait une attitude envers l'Autriche que l'on pourrait qualifier de loyalisme radical, voire soumis. Dans son poème, Hodža fit une déclaration de loyauté envers la patrie autrichienne, il se définit lui-même comme son fils fidèle, il 
souligna le mérite des dirigeants autrichiens, de la l'impératrice Marie-Thérèse et de l'empereur François-Joseph dans le règlement de la situation juridique des paysans. On voit affleurer ici très clairement le mythe du bon empereur répandu dans toute la Galicie, et habilement étayé par la propagande autrichienne.

Le poète présentait l'Autriche comme un territoire pour les Slaves dans leur ensemble, comme la patrie de tous les Slaves. Mais surtout, il soulignait fortement sa signification pour les Slovaques. Le Slovaque «tué » dans le royaume de Hongrie, « ressuscitera » en Autriche. Sa vision de la réorganisation du territoire européen s'opposait clairement avec les projets panslavistes qui plaçaient leurs espoirs en la Russie. Hodža en appelait au concept dominant de "Všeslavi », c'est-à-dire des "slaves dans leur ensemble ", sur lequel se basaient diverses variantes de l'idée slave, et liait cette conception à l'Autriche. En appelant les Slaves à se relever de leur chute, à renaître, il montre l'Autriche comme étant le lieu «de tous les Slaves » (v Rakusii mas tiež Všeslaviu : "en Autriche aussi, tu trouves tous les slaves »). Une fois encore, il tirait sa conception de la tradition grandemorave, associée à celle de Cyrille et Méthode tout en se limitant cependant à sa version mythifiée. Hodža en soulignait deux aspects. Il considérait la Grande-Moravie comme, d'une part, le territoire qui fut le fondement (l'embryon) de la communauté slave, et d'autre part comme le lieu de la mémoire à partir duquel se répandit la mission chrétienne de Cyrille et Méthode.

Dans la construction de cette communauté slave considérée comme une partie de la communauté européenne, le poète assignait un rôle particulier aux Slovaques. Ce rôle provenait du fait qu'ils étaient les héritiers de la tradition grande-morave et qu'ils étaient une nation qui avait souffert, ce qui avait poussé Dieu à les choisir pour réaliser son plan. Cet argument principal était accompagné d'autres, qui s'inscrivaient dans le problème toujours vivace des relations tchécoslovaques. Celles-ci avaient été fondamentalement modifiées après la codification de la langue slovaque. Hodža rappelait que c'était des représentants de la nation slovaque, Pavel Jozef Šáfárik, Ján Kollár et Ján Hollý qui avaient été les premiers à formuler des idées concernant la communauté slave. À cette occasion, il en appelait aux Tchèques pour qu'ils acceptassent, pour le bien de cette communauté, la particularité de la langue slovaque.

Dans la construction de sa conception de la communauté européenne idéale, Hodža n'oubliait pas les questions délicates, celles qui exigeaient d'être résolues au préalable. À côté des relations slovaco-tchèques, celles qu'entretenaient les Slovaques avec les Hongrois méritaient qu'on y prêtât attention. Sa conception d'une patrie slave commune créée dans le cadre de l'Autriche ne s'élevait pas uniquement à l'encontre des projets panslavistes mais également contre l'idée, chère aux Hongrois, d'un État national indépendant qui limiterait le droit national et linguistique des Slovaques (ainsi que des autres nations non hongroises placées dans le périmètre du royaume de Hongrie). Hodža s'opposait très violemment à cette conception politique de la nation, car il voyait en elle la manifestation de ce que l'on appellerait actuellement un colonialisme pour le moins injustifié. Dans un geste chrétien d'unification et de pardon, il considérait cependant que les Hongrois pécheurs avaient une place dans l'Europe transformée.

24 L'utopie messianique qu'exprima Hodža considérait donc les Slovaques, et plus largement les Slaves, comme une nation choisie pour faire renaître l'Europe, elle devait par la force des choses influer sur la situation des autres nations européennes qui devaient quitter l'arène de l'Histoire. Contrairement à la majorité des projets slavophiles ou panslavistes, le poète n'exposait pas une opposition entre Slaves et Européens, il n'opposait pas 
frontalement un Occident « déchu » à un jeune Orient en expansion, il considérait plutôt le Slave comme un élément de l'Europe et l'Europe occidentale vieillissante comme un territoire qui demandait défense et soutien. Il est vrai qu'il reprochait à l'Europe son attitude de colonisatrice envers les nations slaves, mais il tentait aussi de la justifier par le fait que l'Europe ne comprenait pas les vraies valeurs que possédaient ces nations. À la liste des traits communs qu'il assignait aux Slaves, il ajoutait leur rôle de remparts du christianisme. Cet argument lui servait à légitimer la communauté élargie formée par les Slaves et le reste de l'Europe, née de la volonté de Dieu dans le but de s'opposer à la dégradation causée par l'Islam. C'est dans ce contexte qu'apparaissait aux yeux du poète la valeur fondamentale des Slaves : la foi remplaçait chez eux les systèmes philosophiques en vigueur. Nous revenons ici à un problème déjà évoqué qui est le fait que Hodža basait tout son projet historiosophique sur sa foi dans le Christ. Puisqu'ils étaient ceux qui croient en la religion du Christ, les Slaves construiraient une nouvelle communauté, une « Nouvelle Jérusalem ». La Figure du Christ, incarnation du verbe divin, devait devenir un modèle pour les nations appelées à renaître. Elle était un modèle pour le poète lui-même, qui s'imaginait être la nouvelle incarnation du verbe, l'homme de Dieu, auquel fut assignée la mission de réveiller les nations slaves et de montrer la voie, indiquée par le Christ, qu'ils devaient suivre pour être sauvés. C'est l'amour et la souffrance, qui faisaient partie de sa vie, qui le prédestinaient pour ce rôle.

L'historiographie de Hodža se rapproche de la variante polonaise de la pensée messianique, telle que l'incarne Towiański, en ceci que son prophétisme unilatéral expose le rôle du Christ et que la philosophie de l'histoire présentée dans le poème se base sur un fondement fortement religieux. Dans sa typologie du messianisme polonais, Adam Sikora décrit ce type de messianisme comme un messianisme de la foi ${ }^{14}$. Les deux penseurs se retrouvent également sur d'autres conceptions telles que l'idée du développement historique en trois temps et la critique de «l'Église institutionnalisée ». Cette dernière a été, comme on le sait, présentée dans ses cours au Collège de France (1840-1844) par Mickiewicz qui, à différents moments de sa conception historiosophique évolutive, lia le messianisme individuel, personnel, au messianisme national. De multiples trames de «Vieroslavín "vont dans le sens de la pensée de Towiański et de Mickiewicz, on ne peut pas exclure non plus que les conceptions de Jozef Hoene-Wroński ${ }^{15}$ (1778-1853) étaient connues de Hodža. Ce thème s'inscrit toutefois déjà dans la problématique plus large des inspirations polonaises du messianisme slovaque.

Nous avons présenté rapidement, par la force des choses, comment un des messianistes slovaques, qui était d'ailleurs assez peu connu comme tel, considérait sa nation. Ses conceptions jettent une lumière nouvelle sur la position des poètes et des penseurs slovaques au sujet de leur vision du futur et du rôle des Slaves. Dans les conceptions historiosophiques, comme dans les conceptions messianistes (notamment celles de Hroboň, surtout dans sa composition poétique intitulée "Slovenské iskrice») ou missionistes (dont «Slovanstvo a svet budúcnosti ${ }^{16}$ de Štur est un bon exemple) qui se cristallisèrent après la révolution, la mission des Slaves était rattachée au rôle directeur de la Russie. Hodža quant à lui situait son projet dans un autre horizon politique en voyant le futur des Slaves dans l'orbite autrichienne. Et ce après l'accord austro-hongrois de 1867 qui fit de la monarchie des Habsbourg un double État, au moment où même František Palacký, le créateur de l'austroslavisme, croyait de moins en moins à la 
réalisation de ses propositions et qu'il écrivait sa célèbre phrase : « Nous [= les Tchèques] étions là avant l'Autriche, nous seront encore là après elle ", lui qui avait auparavant déclaré que si l'Autriche n'existait pas, il faudrait l'inventer.

\section{NOTES}

1. Pišút M., «Mesianistická báseň Sama Bohdana Hroboňa "Slovenské iskrice” " ( Slovenské iskrice », un poème messianique de Samo Bohdan Hroboň), in : «SBORNIK MATICE SLOVENSKEJ XIX ", 1936, p. 390.

2. Voir Čepan O., Premeny ducha a predmetnosti v slovenskom romantizme (Les changements d'esprit et de réalité dans le romantisme slovaque), in : «LITTERARIA», $n^{\circ}$ XVI : Literárny romantizmus, Bratislava, 1974, p. 102.

3. Nous pouvons également considérer comme correcte l'opinion du chercheur slovaque Oskár Čepan, qui associe l'appréciation du messianisme aux réductions et aux altérations que l'on rencontre dans tout le romantisme slovaque. La raison est à chercher dans ce que les générations ultérieures de critiques s'identifièrent à l'argumentation des romantiques qui s'étaient fixé comme but de ne pas affaiblir les forces nationales. C'est ainsi qu'est né un jugement positiviste et réaliste au sujet du romantisme, selon lequel seuls les actes "constructifs » de la génération romantique sont considérés comme positifs. Voir Čepan O., Op. cit., p. 102.

4. Voir la lettre de Jozef Viktorin, l'éditeur de l'almanach Concordia, à qui Hroboň envoya son "Slovenské iskrice». L'auteur n'y cache pas sa crainte de la censure en ce qui concerne la résonance panslaviste de sa poésie. Čepan cite cette lettre dans son article "Romantický mesianismus a Samo Bohdan Hroboň » (Le messianisme romantique et S. B. Hroboň), in: K problematike slovenského romantizmu (De la problématique du romantisme slovaque), Matica Slovenká, Martin, 1973, p. 102.

5. Le titre est un néologisme forgé par l'auteur du poème, il signifie " celui qui vient de la mère », « la famille issue de la mère ». Le héros de l'œuvre, le brigand Juraj Jánošik, est le « fils de la mère ». Celle-ci représente l'humilité et le pardon, en opposition au personnage du père qui est le protecteur de la vengeance.

6. J'ai consacré une monographie aux transformations du mythe de Jánošik: Goszczyńska J., Mit Janosika $w$ folklorze i literaturze słowackiej XIX wieku (Le Mythe de Jánošik dans le folklore et la littérature slovaque du XIX ${ }^{e}$ siècle), Uniwersytet Warszawski, Instytut Filologii słowiańskiej, Varsovie, 2001, $302 \mathrm{p}$. Cet ouvrage a été traduit en slovaque sous le titre mytus o Jánošikovi vo folklóre a slovenskej literature 19. storočia, JUGA, Bratislava, 2003 (trad. du polonais par Bogumił Suwar).

7. L'attitude prise par les Slovaques durant la révolution de 1848 trouve ses racines dans le conflit qui les opposait aux Hongrois et qui s'était intensifié durant les années 1840. Deux idées s'opposaient : celle du mouvement national slovaque qui entrait à cette époque dans une phase de cristallisation de son programme politique et considérait le royaume de Hongrie comme une patrie commune, et celle du mouvement national des Hongrois qui voulaient transformer leur État multiethnique en État national. L'unification de la langue, décidée par décret en 1840, devait servir ce but: la langue hongroise devait être utilisée non seulement dans les écoles et dans l'administration, mais également dans les églises. 
8. Il est à l'origine, avec Štúr et Jozef Miloslav Hurban, d'un projet de codification de la langue slovaque (1843) ; en 1844, il fut le cofondateur de l'association Tatrín; il fut, en mai 1848, l'un des initiateurs des «Žiadosti slovenského národa» (Les Demandes de la nation slovaque), une pétition destinées aux autorités viennoises par laquelle les Slovaques se déclaraient pour l'égalité des droits avec les Hongrois et la délimitation d'un territoire propre au sein du royaume de Hongrie.

9. Hroch M., V národnim zájmu. Požadavky a cile evropských národních hnutí devatenáctého století $v$ komparativní perspektivě (Dans l'intérêt national. Revendications et buts des mouvements nationaux du XIX ${ }^{\mathrm{e}}$ siècle selon une approche comparative), NLN, Prague, 1995, p. 98 et suiv.

10. Voir Vongrej P., Vyhnanec. Prípad Michal M. Hodža (L'Émigré. Le cas de M. M. Hodža), Spolok slovenských spisovateL'ov, Bratislava, 2000, p. 39.

11. Un choix de fragments tirés de la première partie de l'œuvre, un quart du total environ, fut publié au début du XXe siècle dans le périodique «SLOVENSKÉ POH'ADY ».

12. C'est une partie du dicton hongrois : Kása nem etel, taliga nem szekér, tót nem ember (La kacha n'est pas un repas, une charrette à deux roues n'en est pas une, le Slovaque n'est pas un homme). Voir Hensel L., "Przebudzenie narodowe Słowaków» (Le réveil national des Slovaques), in: Przemiany fomuły polskości $w$ drugiej połowy XIX wieku (Les modifications du concept de polonité durant la seconde moitié du XIX ${ }^{\mathrm{e}}$ siècle) (Sous la direction de Maciejewski J.), Instytut Badań Literackich, Varsovie, 1999, p. 291.

13. Andrzej Findor présente ces relectures dans son article « Tisicročná poroba? » (Une servitude millénaire ?), in : Mýty naše slovenské (Nos mythes slovaques) (Sous la direction de Krekovič E., Mannová E. \& Krekovičová E.), AEP, Bratislava, 2005, pp. 71-76.

14. Sikora A., Myśliciele polskiego romantyzmu (Les Penseurs du romantisme polonais), Verba, Chotomów, 1992, p. 10.

15. Le but recherché par Hœne-Wroński était la réforme du savoir humain et l'établissement de la vérité absolue sur la terre. Ce penseur extrêmement fécond en terme de publications se dit l'inventeur du concept de « messianisme ». (NdT)

16. En français: "Les Slaves et le monde de l'avenir» (milieu des années 1850). Ce texte fut d'abord écrit en allemand puis traduit en russe, il fut publié dans cette langue en 1867. Les œuvres des Hroboň et celles de Štúr ont tant de points communs dans leur expression idéologique que l'on peut les considérer comme des variantes poétiques et publicistes de la fascination qu'exerçait le panslavisme. À ceci près toutefois que le traité de Štúr propose un projet historiosophique élargi et montre d'assez larges convergences avec les idéologues russes de l'époque de l'empereur Nikolaj I, alors que l'œuvre de Hroboň se concentre principalement sur le rôle de la Russie dans la future renaissance des Slaves qui doivent, selon la mission que Dieu leur a assigné, faire renaître l'humanité. Je développe ce thème dans Synowie Słowa. Myśl mesjanistyczn $w$ słowackiej literaturze romantycznej (Les Fils du Verbe. La pensée messianique dans la littérature romantique slovaque), Wyd. Uniwersytetu Warszawskiego, Varsovie, 2008, 220 p.

\section{INDEX}

Mots-clés : histoire de la Slovaquie, littérature slovaque, messianisme

Index chronologique : romantisme, XIXe siècle

Index géographique : Empire austro-hongrois, Slovaquie 


\section{AUTEURS}

\section{JOANNA GOSZCZYŃSKA}

Professeur, directrice de l'Institut de Slavistique (Europe centrale et méridionale) de l'Université de Varsovie (Pologne) 